\title{
Coxoauricular syndrome
}

INSERM

\section{Source}

INSERM. (1999). Orphanet: an online rare disease and orphan drug data base.

Coxoauricular syndrome. ORPHA:1508

Coxoauricular syndrome is an extremely rare primary bone defect, described only in a mother and her three daughters to date, characterized by short stature, hip dislocation, minor vertebral and pelvic changes, and microtia with hearing loss. There have been no further descriptions in the literature since 1981. 